\title{
Germanica
}

\section{Angstbesetzt ist das Leben, Überleben ist die Kunst. Zum Begriff der Heiterkeit in Herta Müllers Collagen}

La vie est tissée de peur, survivre relève de l'art. De la notion d'allégresse dans les collages de Herta Müller

Life is terrifying, the art is to survive. The notion of serenity in Herta Müller's collages

\section{Ute Weidenhiller}

\section{OpenEdition}

Journals

Édition électronique

URL : http://journals.openedition.org/germanica/5454

DOI : 10.4000/germanica.5454

ISSN : 2107-0784

Éditeur

Université de Lille

Édition imprimée

Date de publication : 31 décembre 2018

Pagination : 163-178

ISBN : 978-2-913857-42-1

ISSN : 0984-2632

Référence électronique

Ute Weidenhiller, „Angstbesetzt ist das Leben, Überleben ist die Kunst. Zum Begriff der Heiterkeit in Herta Müllers Collagen", Germanica [Online], 63 | 2018, Online erschienen am: 01 Januar 2021, abgerufen am 22 Januar 2021. URL: http://journals.openedition.org/germanica/5454 ; DOI: https:// doi.org/10.4000/germanica.5454 


\title{
Angstbesetzt ist das Leben, Überleben ist die Kunst. Zum Begriff der Heiterkeit in Herta Müllers Collagen
}

\author{
Ute WEIDENHILLER \\ Università degli Studi Roma Tre
}

\section{Prämissen zu Herta Müllers Schreiben}

Herta Müllers Werk ist wesentlich vom Thema der Angst, von ihren Ursachen und Auswirkungen bestimmt. Staatliche Repressionen in Form von Bespitzelung, Verleumdung, Verfolgung und Todesdrohung sind Erfahrungen, die die Schriftstellerin bekanntlich selbst erleiden musste. Ihr Schreiben, das sie in Anlehnung an Georges-Arthur Goldschmidt ausdrücklich als ,autofiktional', d.h. als vom wirklichen Leben inspiriert betrachtet, entstand aus unmittelbarer Betroffenheit, aus Verzweiflung und Beschädigung, die sich zu Worten formten. „Herta Müller schreibt nie gerne Bücher. Sie schreibt Bücher, wenn sie sich anders nicht mehr zu helfen weiß1." So bringt ihr Freund und Kollege Ernest Wichner die Notwendigkeit der Autorin, ihre Bedrängnisse zu Papier zu bringen, auf den Punkt. Die Schriftstellerin selbst bezeichnet das Schreiben als

1. - Herta Müller - ein Portrait. Ein Film von Angelika Kellhammer (2013) Teil 1 (https://www.youtube.com/watch?v=HhohIgOuhJg, aufgerufen am 8.09.2018). 
„große Belastung“, weil man sich „den Dingen ja dann auch immer wieder stellen" ${ }^{2}$ müsse.

Auf den wiederholten Vorwurf der Kritik, dass es nach all den Jahren - Müller konnte im Jahr 1987 nach Westberlin emigrieren - doch an der Zeit sei, sich neuen Themen zuzuwenden, reagiert die Schriftstellerin mit Empörung:

Manchmal möchte ich laut fragen: Schon mal was gehört von Beschädigung? Von Rumänien bin ich längst losgekommen. Aber nicht losgekommen von der gesteuerten Verwahrlosung der Menschen in der Diktatur, von ihren Hinterlassenschaften aller Art, die alle naselang aufblitzen. Auch wenn die Ostdeutschen dazu nichts mehr sagen und die Westdeutschen darüber nichts mehr hören wollen, läßt mich dieses Thema nicht in Ruhe. Ich muß mich im Schreiben dort aufhalten, wo ich innerlich am meisten verletzt bin, sonst müßte ich doch gar nicht schreiben ${ }^{3}$.

Zur Sprache bringt die Autorin die sie umtreibenden Themen in ihren Romanen und Erzählungen, ihren Essays, Interviews und in besonders knapper und gleichzeitig spielerischer Form in ihren Collagen.

Wie lässt sich nun der Begriff der Heiterkeit mit diesen, hier knapp skizzierten Prämissen vereinbaren bzw. ist Heiterkeit in einem von Angst und Furcht geprägten Alltag überhaupt möglich? Und vor allem: Kann und darf Literatur, die Zeugnis geben will von den Verhältnissen des Alltags in einem diktatorisch regierten Land, heiter sein, und wie kommt eine solche Heiterkeit literarisch zum Ausdruck?

Während erstere Fragen, die sich auf ein allgemeines Verständnis von Heiterkeit als einem Gemütszustand beziehen, relativ klar und einfach beantwortet werden können, bedürfen letztere einer Definition dessen, was unter einer, heiteren' Kunst, unter Heiterkeit im Sinne einer der Kunst immanenten ästhetischen Kategorie zu verstehen ist.

\section{Heiterkeit als Gemütszustand in einem diktatorisch regierten Land}

Momente der Heiterkeit und des Lachens, so erzählt die Autorin, gab es nur im Privaten, im Freundeskreis. Solche Momente bezeichnet die Autorin als überlebensnotwendiges „Glück“ oder auch als „Unfug“:

Die Angst war weggescheucht, wir lachten uns frei. Das war Glück. Vielleicht ein angstgetriebenes Glück, aber ganze Stunden lang. Von

2. - Herta Müller, Von der Macht der Sprache (Sternstunde Philosophie, 2.1.2011, https://www.youtube.com/watch?v=WvCR8JEoExI, aufgerufen am 8.09.2018).

3. - Herta Müller, „Bei uns in Deutschland“, in: Dies., Der König verneigt sich und tötet, Frankfurt a.M., Fischer, 200955, S. 176-185, hier S. 185. 
außen hat es uns geschadet, aber wir wussten ja nicht, dass wir Tag und Nacht abgehört werden und alle Räume unserer Wohnung verwanzt sind. [...] Das Unglück hatte Gründe, die wir alle kannten. Und keiner hing von uns selber ab. Darum brauchte man für das Glück keine Gründe, außer diesem Gespür, dass man dringend darauf angewiesen war. Dieses Glück lebte von seiner Plötzlichkeit und es war notwendiger Unfug 4 .

An anderer Stelle definiert Müller die Beschaffenheit eines ,in den privaten Nischen“" auftauchenden Glücks, ,wo die Schikanen noch nicht eingesickert waren“, noch präziser als ein ,sprunghaftes, eiliges und deshalb überspanntes, ja entfesseltes [...], ein diebisches, ein hinkendes

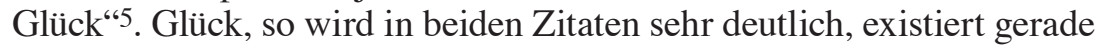
in seiner Dialektik zum Unglück, es wird ausdrücklich ,gegen die Schikanen“ gesucht und muss dem Verrat überlegen sein: „Dieses Glück brauchte so leichte Füße wie der Verrat, weil es ihm davon laufen oder sogar vorauslaufen mußte"6.

Von den vielerlei Arten des Glücks, dem Glück als Zufallsglück, der abergläubischen Glücksbesessenheit der einfachen Leute und dem staatlich verordneten, Glück‘ ist sowohl in Müllers Berichten über ihr eigenes Leben als auch in ihrem schriftstellerischen Werk die Rede. Deutlich tritt in ihrem Roman Herztier (1994) und vor allem in Heute wär ich mir lieber nicht begegnet (1997) hervor, dass all diese Facetten des Glücks dem ,wahren' Glück, das vor allem in der Freiheit verankert ist, diametral entgegenstehen. Es ist meist nur ein ,,verkehrtes Glück“7, das mit einem Heiterkeit schaffenden, unbeschwerten Glück nichts gemeinsam hat. Angst und Verdacht dringen in alle Ritzen des privaten Lebens und gefährden die intimsten Beziehungen. Dass es ,zumindest ein Unglück mit Wünschen"8 darstellt, ist nur ein schwacher Trost, aber immerhin ein Hinweis auf das hartnäckige und verzweifelte Glücksstreben, auf den Versuch, das Glück auch in wenigen Augenblicksmomenten aufzuspüren. Im Roman Atemschaukel (2009) wird zudem klar, wie das Bemühen um eine genaue Definition dessen, was Glück unter schwersten existentiellen Bedingungen bedeuten kann ${ }^{9}$, gleichzeitig Erleichterung

4. - Herta Müller, Mein Vaterland war ein Apfelkern. Ein Gespräch mit Angelika Klammer, München, Hanser Verlag, 2014, S. 95-121, hier S. 115.

5. - Herta Müller, Cristina und ihre Attrappe oder Was (nicht) in den Akten der Securitate steht, Göttingen, Wallstein Verlag, 2009, S. 12.

6. - Ebd.

7. - Das Motiv des, verkehrten Glücks“ zieht sich leitmotivisch durch den Roman Heute wär ich mir lieber nicht begegnet.

8. - Peter Eichhorn, Kritik der Heiterkeit. Ist unserer Literatur die Heiterkeit vergangen?, Heidelberg, Verlag Lambert Schneider, 1973, S. 71.

9. - Zum Thema ,Glück' im Werk Herta Müllers vgl. Ute Weidenhiller, „, Über das Glück nichts, sonst ist es keines mehr'. Von der Paradoxie des Glücks bei Herta 
und daher ein sogenanntes „Kopfglück“10 mit sich bringt. Die Flucht in die Sprache schafft Distanz zur unmittelbaren Bedrohung. In ihrem Essay „Jedes Wort weiß etwas vom Teufelskreis“ erzählt Herta Müller, wie sie sich einst durch das Schreiben vor Einsamkeit und Angst retten konnte:

Ich reagierte auf die Todesangst mit Lebenshunger. Der war ein Worthunger. Nur der Wortwirbel konnte meinen Zustand fassen. Er buchstabierte, was sich mit dem Mund nicht sagen ließ. Ich lief dem Gelebten im Teufelskreis der Wörter hinterher, bis etwas so auftauchte, wie ich es vorher nicht kannte ${ }^{11}$.

Der kreative Umgang mit Sprache wurde unter eingeweihten Freunden zum Gesellschaftsspiel: „Wenn wir zusammen waren, fiel uns allerhand ein. Wir erzählten uns die neuesten Witze, wir spielten stundenlang mit gereimten Sätzen, wir sangen Ceauşescus Reden aus der Zeitung wie Operetten. Reimen und Singen unterwandert den Schädel" ${ }^{\prime \prime 2}$.

In besonders schwierigen Momenten, etwa direkt vor oder nach einem Verhör, wurde das leise Aufsagen von Gedichten oder das eigene Reimen und Erfinden im Kopf zur Gelegenheit, das Denken umzuleiten, um neben sich selbst stehen zu können. Auf welche Weise sich die unerträgliche Realität mittels einer eigenwilligen, widerständigen Vorstellungskraft in die Fantasie rettet und sich Bilder zu einer abstrakten Welt und zu Sprache formen, beschreibt die Schriftstellerin in ihrem Essay „Bei uns in Deutschland“:

Einmal nahmen zwei Männer vom Geheimdienst gleichzeitig ihre Pelzmützen ab, als sie in die Fabrik kamen, um mich zu drangsalieren. Als die Hüte gezogen waren, standen die Haare auf beiden Kopfmitten struppig nach oben. Das Hirn hatte die Haare hochgestellt, um den Kopf zu verlassen - ich sah es, es hockte in der Futterseide.

Müller", in: Martina Wernli/Jens Deeg (Hrsg.), Herta Müller und das Glitzern im Satz. Eine Annäherung an Gegenwartsliteratur, Würzburger Beiträge zur Deutschen Philologie, Bd. 42, Würzburg, Königshausen \& Neumann, 2016, S. 73-91; Dies., „Heute wär ich mir lieber nicht begegnet“ ; „Glück“, in: Norbert Otto Eke (Hrsg.), Herta Müller Handbuch, Stuttgart, Metzler Verlag, 2017, S. 50-58; S. 236-240.

10. - Herta Müller, Atemschaukel, München, Hanser Verlag, 2009, S. 245ff. Vom „Kopfglück“ spricht die Schriftstellerin auch in ihrem Essay „Die Insel liegt innen - die Grenze liegt außen“. (H. Müller, „Die Insel liegt innen - die Grenze liegt außen“, in: Dies., Der König verneigt sich und tötet, Frankfurt a.M., Fischer Taschenbuchverlag, 20094, S. 171f.)

11. - Herta Müller, „Jedes Wort weiß etwas vom Teufelskreis“, in: Dies., Immer derselbe Schnee und immer derselbe Onkel, München, Carl Hanser Verlag, 2011, S. 7-21, hier S. 18.

12. - H. Müller, Mein Vaterland war ein Apfelkern..., a.a.O., S. 114. 
Die beiden Geheimdienstmänner benahmen sich verächtlich, arrogant - nur angesichts der weißen Futterseide waren sie erbärmlich hilflos. Ich fühlte mich unantastbar durch das weiße Glänzen. Ich konnte mich ihnen entziehen, kriegte helle freche Gedanken und sie merkten nicht, was mich schützt. Mir fielen kleine Gedichte ein, ich sagte sie im Kopf auf, als lese ich sie aus Futterseide. Und ihre Hälse schienen alt, ihre Wangen abgenutzt - es war unerlaubt klar, als die beiden Herren über meinen Tod sprachen, daß sie ihrem eigenen nicht werden standhalten können. Wo meine Gedichtchen in der weißen Seide standen, waren ihre beiden Köpfe aufgebahrt ${ }^{13}$.

Herta Müllers sogenannter,fremder Blick' schafft in dieser ausführlich zitierten Passage den Übergang von einer konkreten, äußerst bedrängenden Situation $\mathrm{zu}$ einer abstrakten, realitätsentfremdeten Gedankenfreiheit, die das Gefühl der Überlegenheit gewährt und es somit ermöglicht, der Angst standzuhalten. Die Kunst widersetzt sich dem Tod und überlebt ihn. Die Gewissheit, sich durch Kunst der bedrohlichen Realität entziehen zu können, schafft auch in tiefster Bedrängnis den für eine gewisse Heiterkeit nötigen Abstand. Deutlich wird in der Metapher des ,weißen Glänzen[s]“ Müllers Konzept des konstruktiven Potentials der Sprache, das sie auch als „Trick mit der Sprache“14 oder „Pantomime der Wörter"15 bezeichnet. Einzelne Gefühle und sogar ganze Geschichten verdichten sich zu einem einzigen Wort und lassen gleichzeitig die Realität zu einem dehnbaren Begriff werden. Davon, dass sich ebenso mit wenigen, zum Teil scheinbar zufällig aneinander gereihten Wörtern die aus ihren Erzählungen und Romanen bekannte Welt erzählen lässt, zeugen ihre Wort-Bild Collagen, die kürzeste und zugleich vielseitigste Form ihres Schreibens.

\section{Herta Müllers Kunst der Collage}

Herta Müllers Collagen sind zum größten Teil in den Sammlungen Der Wächter nimmt seinen Kamm. Vom Weggehen und Ausscheren (1993), Im Haarknoten wohnt eine Dame (2000), Die blassen Herren mit den Mokkatassen (2005) und Vater telefoniert mit den Fliegen (2012) erschienen. Eine Ausgabe mit Collagen in rumänischer Sprache wurde 2005 mit dem Titel Este sau nu este Ion veröffentlicht.

Begonnen hat das Aus- und Zerschneiden von oft bunten Wörtern und Bildern, manchmal auch nur Wort- bzw. Bildsegmenten, aus

13. - Herta Müller, „Bei uns in Deutschland“, in: Dies., Der König verneigt sich und tötet, a.a.O., S. 19.

14. - H. Müller, „Immer derselbe Schnee und immer derselbe Onkel“, in: Dies., Immer derselbe Schnee..., a.a.O., S. 96-109, hier S. 101-102.

15. - H. Müller, „Jedes Wort weiß etwas vom Teufelskreis“, in: Dies., Immer derselbe Schnee..., a.a.O., S. 18. 
unterschiedlichen Zeitschriften und das spielerische Zusammensetzen und ungewöhnliche Neukombinieren mit der Idee, Freunden originelle, selbstgebastelte Postkarten zu senden. Diese Arbeiten implizieren einen völlig neuen und anderen, sinnlichen und experimentellen Umgang mit Sprache. Es geht der Schriftstellerin um die Montage bzw. das Zerlegen und Wiederaufbauen von Begriffen, d.h. um das Nachforschen und Verstehen des Funktionierens von Sprache und um deren Materialcharakter, der dabei optisch wirksam miteinbezogen wird.

Der Anfang jeder Collagekarte entsteht hauptsächlich aus dem Zufall, um dann in eine bestimmte Sinnrichtung gelenkt zu werden. Die meist surreal arrangierten Bildschnipsel - es handelt sich um Fragmente von Landschaften, Gegenständen oder Körper, um Schattenbilder oder Zeichnungen - sind zusammen mit dem mehrfach gebrochenen ${ }^{16}$, sich z.T. reimenden Wortmaterial so kunstvoll arrangiert, dass durch die Kombination von Optik, Klang und Rhythmus aus jeder Gedichtcollage ein Gesamtkunstwerk entsteht. Dem Leser präsentiert sich eine groteske und oft spiegelverkehrte Welt, feindlich gegenüber der Gemeinschaft wie auch dem Einzelnen.

Unhaltbar ist der Vergleich mit dadaistischen Kunstwerken oder mit Nonsensdichtung, denn jede Collage, so betont Müller, muss einen Inhalt haben und etwas Konkretes erzählen. Nervosität und Angst, staatliche Überwachung und Bedrohung, Verrat und Lüge, Mord und Tod, Flucht und Heimweh, Einsamkeit, Identitätsverlust und Beschädigung sind die vorherrschenden Themen der Collagen. Gleichzeitig geht es um die Suche nach neuen und ungewöhnlichen Wortkombinationen, die geeignet sind, all diese Erfahrungen so anschaulich wie möglich zu vermitteln. Einzelne, u.a. den Staat vertretende Akteure der Collagen, wie etwa Mutter und Vater, Nachbar und Nachbarin, der Friseur, der Wächter, der Polizist oder der Mann vom Geheimamt, der Gerichtsmediziner, die toten Freunde und nicht zuletzt der unter der Chiffre König oder Frosch auftauchende symbolisch verschlüsselte Diktator sind dem eingeweihten Leser ebenso vertraut wie die Landschaften, Schauplätze und Atmosphären, vor denen sie erscheinen: Das Dorf, das grüne Tal, die gelben Mais- und Weizenfelder, der Wind und vor allem der Schnee ${ }^{17}$, bestimmte Bäume und Pflanzen wie die Pappeln, der Aprikosenbaum

16. - Herta Müller, „Die Anwendung der dünnen Straßen“, in: Dies., Immer derselbe Schnee..., a.a.O., S. 110-124, hier S. 114f.

17. - Der Schnee ist direkt mit dem Thema des Verrates verbunden. Im Essay „Immer derselbe Schnee und immer derselbe Onkel“ beschreibt Herta Müller, wie der Schnee die Fußspuren ihrer Mutter sichtbar machte und dadurch ihre Zwangsdeportation ins Arbeitslager verursachte. Auch in Vater telefoniert mit den Fliegen spielt das Motiv des Schnees und die mit ihm verbundene Farbe Weiß eine zentrale Rolle. 
oder die Dahlie, auch die Grenze, die Stadt, der Asphalt sowie für Herta Müller symbolische Gegenstände, etwa der Koffer oder das Taschentuch und bestimmte Farben evozieren Assoziationen, die zu ihren erzählerischen und essayistischen Schriften zurückführen. Vielseitig sind nicht nur die Collagen selbst, sondern ebenso die Blickwinkel aus denen einzelne wiederkehrende Themen und Motive beleuchtet werden. Szenarien ihrer dörflichen Kindheit sowie Orte, Täter und Opfer der späteren, in der Stadt erfahrenen staatlichen Repressionen bilden ebenso wie die Ausreise in den Westen den biographischen Hintergrund, vor dem jede einzelne Collage-Arbeit zu verstehen ist. Voraussetzung für das Verständnis dieser literarischen Miniaturen ist die Kenntnis des engmaschigen Netzes von Bedeutungen ${ }^{18}$, das zwischen Müllers Werken besteht. Die Collagen sind ausdrücklich ,eine Art zu schreiben“19, sie bilden unbewusste Vorarbeiten für ihre Prosa und haben im Lauf der Jahre eine immer größere künstlerische Eigendynamik entwickelt:

Aber ich muß auch da in Kürzestform immer etwas erzählen. Da kann ich reimen, unauffällig, damit sich die Sätze diskret binden, es muß unauffällig sein, als hätte es sich so ergeben. Der Rhythmus der Sätze muß da sein. Man muß es laut lesen können, ohne mit der Zunge zu stolpern. Und ich muß das Bild der Sätze im Kopf gesehen haben, um sie auf ihre Plausibilität zu prüfen. Das ist mit den Collagen genauso wie mit der Prosa. Wenn es sich gut anhört und gut anschauen läßt, dann kann es so bleiben ${ }^{20}$.

In der eigenwilligen Kombination von Wörtern und Assoziationen und in der Suche nach Reim, Rhythmus und Takterweisen sich die Collagen als rhetorisch gefeilte, hochartistische Gebilde, die durch den paradoxen Gegensatz zwischen spielerischer Rhetorik und ernstem Inhalt nicht selten eine Art Galgenhumor an den Tag legen. Auf die Frage, warum die Gedichtcollagen oft lustig sind, während den Romanen der Humor versagt zu sein scheint, antwortet die Schriftstellerin: „In meinen anderen Büchern, in denen es um die Diktatur geht, kann ich mir das nicht leisten. Der Humor ist aber eine Seite von mir, im Persönlichen,

18. - Als „rhizomatisches Geflecht wiederkehrender Bilder, Themen und Motive“ bezeichnet Norbert Otto Eke bereits die Postkartensammlung Der Wächter nimmt seinen Kamm und den späteren Band Im Haarknoten wohnt eine Dame. Norbert Otto Eke, „Schönheit der Verwund(er)ung. Herta Müllers Weg zum Gedicht", TEXT+KRITIK Herta Müller, 155/2002, S. 64-79, hier S. 70.

19. - Herta Müller, „Ich habe die Sprache gegessen”. Die LiteraturNobelpreisträgerin Herta Müller über ihre zusammengeklebten Gedichte und über die Macht und das Versagen der Wörter“, Der Spiegel 35/2012, S. 128-132, hier S. 128.

20. - Herta Müller, Lebensangst und Worthunger. Im Gespräch mit Michael Lentz, Berlin, Suhrkamp, 2010, S. 54. 
mit Freunden. Ich hole mir über die Collagen etwas zurück, ich kann den finsteren Themen da anders begegnen ${ }^{21}$.“

Auf unterschiedliche und gegensätzliche Art und Weise kommt der Begriff der Heiterkeit in Herta Müllers Collagen implizit zum Tragen: Wo Heiterkeit als Stimmung und Gefühl nicht wirklich möglich ist, kann sie in der Freiheit des poetischen Spiels als ästhetische Kategorie existieren und damit „Künstler wie Publikum [...] vom Druck der Wirklichkeit" ${ }^{\prime 2}$ entlasten.

\title{
Vermeintliche Heiterkeit, lakonisches Lachen und Hohngelächter
}

Wie prekär und doppelbödig vermeintlich heitere Situationen in einem totalitär regierten Land sind, tritt sehr deutlich in folgender Collage aus der Sammlung Im Haarknoten wohnt eine Dame (2000) zu Tage:

\author{
Wenn der Friseur Akkordeon spielt \\ liegt noch das Messer auf dem Tisch und \\ jedes Lied nimmt sich ein Glück \\ woher es kann \\ das Gefühl wird nur zitiert \\ bloß der Verdacht gleicht einem Kraut das \\ hier gewachsen ist \\ zwei Fotos werden noch gemacht. Im Garten \\ tanzt der Vater glattrasiert und \\ Mutter zögert unter einem Baum und \\ hat ein Lächeln anprobiert das \\ ist geborgt von einem Kalb ${ }^{23}$
}

Die Gefühle, Handlungen und Gesten, die den Rahmen der hier evozierten Szene bilden, sind, so die Botschaft, nicht authentisch, sondern nur noch künstlich von außen aufgesetzt. Das „Glück“ existiert nicht wirklich, es wird lediglich im Lied zitiert, das „Lächeln“ ist keine echte, spontane Gefühlsregung, es ist vorgetäuscht und gleicht einem ausgeliehenen Kleidungsstück, das man ,anprobiert“. Wo der Verdacht, versinnbildlicht durch die Metapher des heimischen Krautes, als vorherrschendes und alles überwucherndes Gefühl dargestellt wird, kann Musik und Tanz keine Heiterkeit mehr schaffen und auch in der

21. - H. Müller, „Ich habe die Sprache gegessen“, a.a.O., S. 128.

22. - Petra Kiedaisch, Ist die Kunst noch heiter? Theorie, Problematik und Gestaltung der Heiterkeit in der deutschsprachigen Literatur nach 1945, Tübingen, Max Niemeyer Verlag, 1996, S. 23.

23. - Herta Müller, Im Haarknoten wohnt eine Dame, Hamburg, Rowohlt, 2000. Die Seiten dieses Bandes sind nicht nummeriert. 
doppelten Konnotation von „Messer“ - als Werkzeug des Friseurs ${ }^{24}$ und gleichzeitig als Waffe - liegt eine unterschwellig lauernde Bedrohung. Fröhlichkeit und Heiterkeit wird in dieser Collage durch die unsichtbare, subtil spürbare Anwesenheit des totalitären Regimes unterminiert. Die Figurenkonstellation von Vater, Mutter und Friseur verweist auf den aus Niederungen (1984) bekannten Familien- und Dorfkontext. Auch die dort herrschenden patriarchalischen Strukturen treten an die Oberfläche, denn während der sich für einen wohl festlichen Anlass zurecht gemachte, frisch rasierte Vater scheinbar unbeschwert tanzt, fügt sich die Mutter nur zögernd und notgedrungen der Situation. Ihr ausdrücklich von einem Kalb geborgtes „Lächeln“ ist als Metapher der in Müllers Werk eingeschriebenen Opferthematik zu verstehen. Das in den Collagen mehrmals evozierte Lachen ist stets Ausdruck negativ besetzter Gefühle, wie etwa in folgender Komposition, in der es um das problematische Thema der Heimat geht:

An der Grenze hat mich der Wachmann mit dem Oberlippenbart gefragt

Warum setzen sie auf Heimat zum

Quadrat? Ich habe ein wenig gelacht

viel an die freien Schuhe der

Fasane an den lauen Schlafsamen

der Nacht an das Muster der

Haut auf der Milch an die

Schönheit der Fältchen der Kälte gedacht

dabei zwei schöne Augen gemacht 25

Was das kurze und lakonische, auf die Frage des Grenzwächters folgende Lachen beinhaltet, lassen die abschweifenden Gedanken des lyrischen Ich erahnen. Es liegt in ihnen die Melancholie der bei Müller im Begriff der Heimat impliziten Unfreiheit des Individuums. Dem Gemütszustand der Heiterkeit diametral entgegengesetzt ist das Lachen in folgender Collage, denn es entspringt einem brutalen Sarkasmus und ist ein reines Hohngelächter über den als Unfall inszenierten, vermutlich im staatlichen Auftrag verübten Mord:

der Elektriker blieb mit den Fingern am Draht

über dem Tabakfeld hängen

es kam Polizei

mit dem blauen Licht

aus dem Rock des Verunglückten

24. - Vgl. dazu Martina Wernli, „Haarige Geschichten. Zur Figur des Friseurs bei Herta Müller", in: Martina Wernli/Jens Deeg (Hrsg.), Herta Müller und das Glitzern im Satz..., a.a.O., S. 193-215.

25. - H. Müller, Vater telefoniert mit den Fliegen, a.a.O., S. 12. 
flogen drei

oder vier wilde Enten ins Gras

man hörte sie lachen

daß es ein Unfall war

oder nicht

\section{Heiterkeit als ästhetische Kategorie - die spielerische Sphäre der Sprache}

Abgesehen von inhaltlichen und begrifflichen Aspekten, die mit dem Begriff der Heiterkeit assoziiert werden können, existiert in Müllers Collagen noch eine ganz andere, eine ästhetische Kategorie von Heiterkeit, die im spielerischen Umgang mit dem kreativen Potential der Sprache zum Tragen kommt:
Am 1. Juli gab es ein Malheur
Mein Vater verwechselte die Flaschen
und trank Kartoffel-Käfer-Gift statt Wein
im Kühlschrank stand auch noch Likör
den trank der Gendarm am Küchentisch
und schrieb uns ein Stück Totenschein
na ja das Leben das kann keine Hure sein
denn der Pförtner bezahlt meine Mutter nicht
er ist blau tätowiert ich bin junger Klee
mir zahlt er pro Stunde 400 Lei. $^{26}$

Eine Art Galgenhumor entsteht hier durch die lapidare Bezeichnung des tragischen Vergiftungstodes des Vaters als „Malheur“. Der Duden definiert das umgangssprachliche bzw. veraltete Wort als ein „nicht sehr folgenschweres Missgeschick, Unglück, das den Betroffenen in eine peinliche Situation bringt". Häufig setzt man dem Begriff das Adjektiv klein voran, um die Unwichtigkeit des Missgeschicks zu unterstreichen. Der Reim sowie der umgangssprachliche, lockere Ton, der im Kontrast steht zu der verzweifelten, von einem weiblichen lyrischen Ich geschilderten Lage, erzeugen beim Leser ebenfalls einen heiteren Effekt. Während unklar bleibt, wie diese fatale Verwechslung zwischen Kartoffel-Käfer-Gift und Wein zustande kam, bzw. wie das Gift überhaupt in den Kühlschrank gelangen konnte, geht eindeutig hervor, dass sich sowohl die Mutter als auch die hier als lyrisches Ich sprechende Tochter prostituieren. Der Verdacht, dass der Staat in beiden Angelegenheiten die Hand im Spiel hat, drängt sich auf, vermuten lassen sich Erpressung und hinterhältiger Mord.

26. - H. Müller, Im Haarknoten wohnt eine Dame, a.a.O. 
Leicht und unbeschwert klingt die Sprache in folgendem Sechszeiler:

$$
\begin{aligned}
& \text { meine liebe Mutter spinnt } \\
& \text { hält den Waschtisch für ihr Kind } \\
& \text { und mein lieber Vater spinnt } \\
& \text { mäht im Garten kahlen Wind } \\
& \text { und ich habe mir gedacht } \\
& \text { gib gut acht wie man es macht }{ }^{27}
\end{aligned}
$$

Das regelmäßige Metrum mit dem Reimschema ababcc, der reine Reim und die simple Sprache machen das Gedicht luftig und eingängig. Die so geschaffene formale Schlichtheit und Durchsichtigkeit gestaltenden schlimmen Inhalt eher lustig und heiter. Der Inhalt steht nicht mehr im Vordergrund, wird als solcher gar nicht mehr wahrgenommen und geht vollkommen in der Form und in der Wortspielerei unter. Wiederholt erzählt die Autorin vom Umleiten der Angst in Reime oder Lieder und am Beispiel einiger Passagen aus Ruth Klügers Autobiographie weiter leben. Eine Jugend gibt sie ihre eigene Strategie zu erkennen. Klüger erzählt dort, wie ihr das Aufsagen Schillerscher Balladen, ,die Form selbst, die gebundene Sprache, eine Stütze gab“ und sie dann eigene Kindergedichte verfasste, ,die in ihrer Regelmäßigkeit ein Gegengewicht zum Chaos stiften wollten [...]“. Denn, so zitiert Müller weiter: „Wer nur erlebt, reim- und gedankenlos, ist in Gefahr, den Verstand zu verlieren [...]." 28

Allgegenwärtige Überwachung der staatlichen Handlanger raubt den Menschen den Verstand und verwandelt Dinge in andere, das ist auch die Kernaussage des oben genannten Gedichts, das im Band Vater telefoniert mit den Fliegen in zwei inhaltlich ähnlichen, im Ton jedoch völlig anderen Versionen erscheint:
Mutter hält den Rauchfang für
eine Wolkenziege und
Vater die Nähmaschine für
ein Pferd und der Herbst läuft
einfach über die Hügel warm
und spiegelverkehrt ${ }^{29}$

Einen komischen Effekt erzielt hier die Absurdität der von Mutter und Vater verwechselten Dinge, wie die in Müllers Werk oft ,,spiegelverkehrt" geschilderte Welt im Allgemeinen. Unheimlich und gleichzeitig

27. - Ebd.

28. - Herta Müller, „II. Sag, dass du fünfzehn bist - weiter leben Ruth Klüger“, in: Dies., In der Falle, Göttingen, Wallstein Verlag, S. 37f.

29. - Herta Müller, Vater telefoniert mit den Fliegen, München, Hanser Verlag, 2012, S. 69. 
grotesk wirkt ebenso die im Bericht eines Mannes vom Geheimamt auftretende Rollenvertauschung zwischen einem Hund und seinem Herrchen:

\author{
Der Mann \\ vom Geheimamt \\ folgt Herrn Frank \\ und meldet am gleichen Tag \\ unter Aktenzeichen 005/2011: \\ 4. Mai Punkt 15 Uhr geht Element \\ Frank mit dem rothaarigen Hund \\ Else in eine Kirche hinein ich \\ verstecke mich in der Ecke sie \\ glauben sich allein Element Frank \\ bellt hastig Hund Else zündet eine \\ Kerze an mir wird es mulmig \\ dann verschwinde $\mathrm{ich}^{\text {“30 }}$
}

Eine Collage über den Zustand von Orientierungslosigkeit und Verwirrung kann aber auch ausnahmslos verzweifelt klingen:
Als ich vom Verhör kam
war ich niemandes Kind mehr
und mit mir nicht mehr verwandt
am Strassenrand liefen die Möbel
der Bäume nur wo kam
der Wind her ${ }^{31}$

Herta Müllers Collagen bilden ein äußerst heterogenes Werk: „Tröstungsfrei, bildkarg, [...] wortgenau und hoffnungslos“32 zum einen, spielerisch, kreativ und vieldeutig zum anderen. Durch das „Detail der Sinne“ und den ,persönlichen Blickfang“ 33 versucht die Schriftstellerin in ihren poetischen Texten dem Leser das ganze Ausmaß der Tragödie eines von der Diktatur bestimmten Lebens anhand einzelner Episoden und Schicksale als sinnlich nachvollziehbar zu schildern. Raffinierte Sprach-Bild Strategien durch exotische Metaphern, Metonymien oder auch hermetische, leitmotivische Symbole führen zu einem dichten Gewebe von Verweisungen und zu ungewöhnlichen Bildern. Die Autorin lenkt den politischen auf einen ästhetischen Pol um und verarbeitet die staatlichen Repressionen auf künstlerisch produktive Art und Weise.

30. - Ebd., S. 22.

31. - Ebd., S. 69.

32. - Jürgen Wertheimer, „Im Papierhaus wohnt die Stellungnahme. Zu Herta Müllers Bild-Text-Collagen“, TEXT+KRITIK, Herta Müller, 155/2002, S. 80-84, hier S. 80.

33. - H. Müller, „I. In der Falle“, in: Dies., In der Falle, a.a.O., S. 5. 
Die von ihr geprägte Chiffrensprache ist Ausdruck des Misstrauens in ein Zusammenhang stiftendes Ganzes, die Wörter für das Beschreiben der sie umgebenden Realität müssen neu erfunden werden:

Nur der Wortwirbel konnte meinen Zustand fassen. Er buchstabierte, was sich mit dem Mund nicht sagen ließ. Ich lief dem Gelebten im Teufelskreis der Wörter hinterher, bis etwas so auftauchte, wie ich es vorher nicht kannte. Parallel zur Wirklichkeit trat die Pantomime der Wörter in Aktion. Sie respektiert keine realen Dimensionen, schrumpft die Hauptsachen und dehnt die Nebensachen. Der Teufelskreis der Wörter bringt dem Gelebten Hals über Kopf eine Art verwunschene Logik bei ${ }^{34}$.

Eigentümliche Sinnverbindungen schafft die Autorin vor allem durch das Stilmittel des Vergleichs. Bizarre, Humor und Heiterkeit nicht entbehrende Bilder verschiedenster Arterscheinen teils als ausdrückliche Vergleiche, teils zur Metapher verkürzt. Die „Ameisenhügelfrisur“35 der Mutter, die „Wolkenziege“"36 in der oben zitierten Collage, das „Gelbrockmuster / des langsam schmal werdenden Mondes“"37 oder das „nierengelbe Maisfeld“ 38 sind nur Beispiele für die zahlreichen Neologismen.

Sprache überschreitet in Müllers Collagen ihre medialen Grenzen. Das ,Bild‘ kann sich in diesem Genre gleichzeitig in seiner ganzen Vielfalt, nämlich als sprachliches, optisches und graphisches Bild entfalten. Dies ist die Konsequenz ihrer Bemühung um Genauigkeit im Beschreiben des Wahrgenommenen bei gleichzeitiger Distanznahme zum wirklich Geschehenen, das die reale Angst im verzerrten und verwandelten Bild zu bannen scheint:

Wenn man im Beschreiben genau sein will, muss man im Satz etwas erfinden, das ganz anders ist, damit man genau sein kann. [...]. Das war vielleicht eine Sucht, diese im Kopf eingebildeten Gegenstände mit ihren vagabundierenden Eigenschaften. Ich suchte sie fortwährend, deshalb suchten sie mich. Sie liefen mir wie eine Meute nach, als würde ich sie mit meiner Angst füttern. Wahrscheinlich fütterten sie mich, gaben meiner Angst ein Bild. Und Bilder, vor allem bedrohliche, müssen nicht trösten, und darum müssen sie nicht enttäuschen, und darum zerbrechen sie nie. Man kann sich immer wieder dasselbe Bild einbilden im Kopf. Durch und durch bekannt, wird es immer zu

34. - H. Müller, „Jedes Wort weiß etwas vom Teufelskreis“, in: Dies., Immer derselbe Schnee..., a.a.O., S. 7-21, hier S. 18.

35. - H. Müller, Vater telefoniert mit den Fliegen, a.a.O., S. 29.

36. - Ebd., S. 54.

37. - Ebd., S. 132.

38. - Ebd., S. 164. 
einem Halt. Die Wiederholung machte es jedesmal wie neu, und sie schonte mich $^{39}$.

Grundlegend für das Verständnis Müllers eigenwilliger Metaphern und das Verfahren ihrer Wortbildung sind einige Essays, in denen sie anhand konkreter Beispiele den Ursprung bestimmter Begriffe kommentiert. In Immer derselbe Schnee und immer derselbe Onkel aus der gleichnamigen Sammlung erklärt die Schriftstellerin ausführlich, wie bestimmte, reale oder der Phantasie entsprungene Bilder die Gegenstände in andere verwandeln und die häufig bizarr anmutenden Sprachbilder ihrer Texte somit aus dem Bemühen um Genauigkeit der Beschreibung entstehen: „Wenn man im Beschreiben genau sein will, muss man im Satz etwas finden, das ganz anders ist, damit man genau sein kann" $" 40$.

„Die Frisuren der Frauen“ verwandeln sich in „sitzende Katzen“, „dünne, im Wasser schwimmende“41 Äste in angsteinflößende Wasserschlangen. Bedarf letzteres Bild keiner näheren Erläuterung, so lässt sich ersteres aus einer bestimmten, traditionellen Haartracht erklären:

Jede Frau im Dorf hatte einen langen dicken Zopf. Doppelt zusammengelegt wurde der Zopf am Hinterkopf senkrecht nach oben geführt und mit einem halbrunden Hornkamm oberhalb der Kopfmitte hochgesteckt. Die Zähne des Hornkamms verschwanden im Haar, von seinem gewölbten Rand schauten nur die Ecken wie kleine, spitze Ohren heraus. Mit den Ohren und dem dicken Zopf sah der Hinterkopf der Frauen wie eine kerzengerade sitzende Katze aus ${ }^{42}$.

Als raffiniertes künstlerisches Bild präsentiert sich vor allem in Müllers jüngster Sammlung Vater telefoniert mit den Fliegen jede einzelne Collage und damit die Sprache selbst. Wörter und Wortsegmente erscheinen auf oft farbigem Hintergrund, sie variieren in Größe und Schriftart und setzen damit bereits bestimmte optische Akzente. Ins Auge stechen in einigen Collagen einzelne Wörter, die aufgrund ihrer besonders auffälligen Graphik ganz offensichtlich aus dem ursprünglich gleichen Text stammen. Der Leser bringt sie bereits bei einem flüchtigen Blick miteinander in Verbindung, erkennt sie als naiv anmutende Sprachspielerei ${ }^{43}$ oder auch als Signalwörter, die nicht

39. - H. Müller, „Immer derselbe Schnee und immer derselbe Onkel“, in: Dies., Immer derselbe Schnee..., a.a.O., S. 97.

40. - Ebd., S. 96.

41. - Ebd.

42. - Ebd.

43. - Wie in einem Wort-Puzzle für Kinder verbinden sich in einer Collage die Bildkarten Kuh und Stall zu einem Wort. Vgl. H. Müller, Vater telefoniert mit den 
selten die Aufmerksamkeit auf die zentrale Aussage oder Struktur des Gedichtes lenken. In der Kombination des spielerisch-heiteren sprachlichen Umgangs mit ernsten Inhalten steckt sprachliche Raffinesse. Wie wichtig die Inhalte dabei bleiben, zeigt Müllers Kritik am unbedachten Umgang mit Sprache, die in folgender Collage wiederum in einen leichten Ton gepackt ist. Ironisch enthüllt ein lyrisches Ich die „Einfachheit“ eines gewissen Herrn Straub anhand seiner Äußerung nichtssagender Werbeslogans, die ihn zu einer lächerlichen Figur werden lassen:

\author{
Gestern sagte Herr Straub \\ Holz macht stolz \\ und dann \\ Was immer passiert, Hauptsache kariert \\ Ich dachte, er nimmt sich \\ Die Einfachheit die \\ es eilig hat, aus dem Silbersack \\ der Zeit ${ }^{44}$
}

In scharfem Ton denunziert die Schriftstellerin in ihrem Essay „Die Falle“ den „Hinterhalt der Worte“, die „Dreistigkeit der Redewendungen und Sprichwörter" 45 und deren unangemessenen Gebrauch im Alltag: „Redewendungen verabsolutieren. Im Deutschen haben sie die Steigerung ins Töten begleitet. Vielleicht sollte man in jeder Sprache, und besonders in der deutschen, ohne diese Treffer auskommen und Worte finden, die im eigenen Mund entstehen" ${ }^{46}$.

Herta Müller war gewohnt an eine knappe, ,geraffte Sprache“47 oder das Schweigen, der sinnentleerte Umgang mit Sprache in Form von leeren Phrasen ist ihr aus der banatschwäbischen Heimat unbekannt. In ihrem Essay „Bei uns in Deutschland“ erzählt sie eine für diesen Kontext beispielhafte Episode. Auf Müllers Erklärung, dass in Rumänien Lammbraten ein Traditionsgericht sei und dass „das rumänische Nibelungenlied, das Nationalepos [...] von Schafen und Hirten“ handele, antwortet ihr Gastgeber „Ist ja lustig“. Müller, die solche Bemerkungen nicht kannte und den Inhalten der Wörter nachging, antwortete daraufhin: „Es ist nicht lustig, es geht um Betrug und völlige Verlassenheit in der Angst, um Schmerz und Tod 48 .“

\footnotetext{
Fliegen, a.a.O., S. 15.

44. - Ebd.

45. - H. Müller, „I. In der Falle“, in: Dies., In der Falle, a.a.O., S. 36.

46. - Ebd., S. 36f.

47. - Herta Müller, „Gelber Mais und keine Zeit“, in: Dies., Immer derselbe Schnee und immer derselbe Onkel, a.a.O., S. 125-145, hier S. 125.

48. - H. Müller, „Bei uns in Deutschland“, in: Dies., Der König verneigt sich und tötet, a.a.O., S. 177.
} 


\section{Resümee}

Die Heiterkeit und das Heitere sind Begriffe, die in Herta Müllers Werk nicht geläufig sind, vielleicht aufgrund der dem Begriff impliziten Eigenschaften positiver Gelassenheit, wohlwollenden Behagens und tröstlicher Versöhnlichkeit, die nur schwer mit den Grundlagen und Themen ihres Schreibens zu vereinbaren sind. Eine Versöhnung49 mit der Vergangenheit, mit ihrer ehemaligen Heimat schließt die Schriftstellerin kategorisch aus:

Was heißt Versöhnung? [...] Ja, womit soll ich mich versöhnen? Man kann sich doch nur mit einzelnen Dingen versöhnen. Ich weiß auch nicht, wie ich das tun sollte. Also mit dem Geheimdienst kann ich mich nicht versöhnen. Was heißt auch versöhnen. Ich will mich nicht versöhnen! Ich kann mich doch nicht versöhnen mit einem Geheimdienst oder mit einem diktatorischen System ${ }^{50}$.

Für die in ihrem Werk jedoch zweifellos wichtige kritische Hinterfragung der Gemütsverfassung und der Wünsche von in Grenzsituationen lebenden Menschen verwendet sie den facettenreicheren Begriff ,Glück', für eine fröhlich aufgelockerte Stimmung auch den Begriff ,Unfug'. Das gewöhnlich als Ausdruck der Heiterkeit geltende Lachen wird ebenfalls erwähnt und - wie gezeigt wurde - ad absurdum geführt.

Die von Petra Kiedaisch in ihrer Untersuchung Ist die Kunst noch heiter? vorgestellte, auf Peter Eichhorns Studie basierende These, dass Heiterkeit ,mit künstlerischen Mitteln in der Literatur konkret herstellbar ist und als eine Gestaltqualität betrachtet werden kann “51 lässt sich für Herta Müllers Werk anhand ihres raffinierten und revolutionären Umgangs mit Sprache bestätigen.

49. - Heiterkeit ist nach Schiller ein „Geschenk der Kunst als eine Gabe des Gottes Apoll, des Musenführers, an den bedürftigen Menschen, dessen Leben ernst, d.h. vom „Antagonismus der Kräfte“ gekennzeichnet. Sie ist so der Zustand des Gemütes, der nach dem Durchgang durch die Entzweiung mit dem Begriff der Versöhnung umschrieben werden kann. Gregor Sauerwald, „Heiterkeit, das Heitere“, in: Joachim Ritter (Hrsg.), Historisches Wörterbuch der Philosophie, Basel, Schwabe Verlag, 1974, Bd. 3, Sp. 1039-1043, hier S. 1040.

50. - Herta Müller: Von der Macht der Sprache (Sternstunde Philosophie, 2.1.2011), a.a.O.

51. - P. Kiedaisch, Ist die Kunst noch heiter?, a.a.O., S. 29. 